2. Seo D, Lacadie CM, Tuit K, Hong KI, Constable RT, Sinha R. Disrupted ventromedial prefrontal function, alcohol craving, and subsequent relapse risk. JAMA Psychiatry. 2013;70:727-39.

3. Hanlon CA, Dowdle LT, Gibson NR, Li X, Hamilton S, Canterberry M, et al. Cortical substrates of cue-reactivity: transdiagnostic relevance of the medial prefrontal cortex. Transl Psychiatry. 2018;8:186.

4. Hanlon CA, Dowdle LT, Henderson JS. Modulating neural circuits with transcranial magnetic stimulation: implications for addiction treatment development. Pharmacol Rev. 2018;70:661-83.
5. Kearney-Ramos TE, Dowdle LT, Lench DH, Mithoefer OJ, Devries WH, George MS, et al. Transdiagnostic effects of ventromedial prefrontal cortex transcranial magnetic stimulation on cue reactivity. Biol Psychiatry Cogn Neurosci Neuroimaging. 2018;3:599-609.

6. Hanlon CA, Dowdle LT, Correia B, Mithoefer O, Kearney-Ramos T, Lench D, et al. Left frontal pole theta burst stimulation decreases orbitofrontal and insula activity in cocaine users and alcohol users. Drug Alcohol Depend. 2017;178:310-7.

\title{
Decoding the role of the microbiome on amygdala function and social behaviour
}

\author{
John F. Cryan $\mathbb{1}^{1,2}$ and Timothy G. Dinan ${ }^{2,3}$ \\ Neuropsychopharmacology (2019) 44:233-234; https://doi.org/10.1038/s41386-018-0233-3
}

We are living in a microbial world with our bodies having as many microbial cells as human cells. Growing evidence implicates these microbes, known collectively as the microbiome, as key regulators of brain function and behaviour [1]. One of the key findings from across many species is that the microbiome affects social behaviour [2]. We have shown that germ-free (GF) mice, which grow up in a sterile environment and thus have no bacteria in or on their bodies, are less sociable than normal mice[2]. Moreover, the amygdala, a brain region important for social behaviour, is particularly sensitive to changes in microbiome composition [3] and GF mice have widespread changes in amygdala neuronal morphology and function [4].

Ongoing research is trying to determine the molecular mechanisms underpinning such effects. Initially, we exploited unbiased genome-wide transcriptional profiling to determine gene expression in the amygdala of male GF mice. We found differential gene expression, exon usage and RNA-editing in GF mice (Fig. 1). We noticed upregulation of several immediate early response genes such as Fos, Fosb, Egr2 or Nr4a1 in association with increased cAMP response element-binding protein (CREB) signalling in GF mice [5]. In addition, we found differential expression and recoding of several genes implicated in a variety of neuronal processes such as neurotransmission, neuronal plasticity, metabolism and morphology. These data strongly suggest altered baseline neuronal activity in the amygdala of GF animals, which may underpin the social deficits. However, what happens under a social stimulus remained known.

To this end we recently described dynamic regulation of several previously undescribed pathways in response to social stimulation. These include regulation of RNA-processing non-coding RNAs that are crucially involved in splicing regulation. Moreover, social stimulus evoked an increase in transcripts of genes involved in neuronal activity, which includes induction of several well established immediate early genes such as Fos or Arc, the MAP-K pathway and neurotrophic signalling via Bdnf. Moreover, we find upregulation of complement components, which have lately been established to be necessary for synaptic rearrangements and plasticity upon neuronal activity [6].

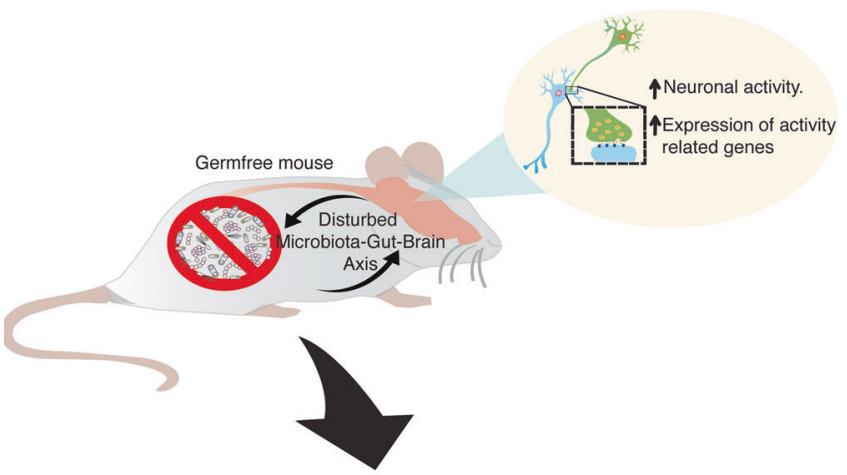

Social interaction behaviour paradigm
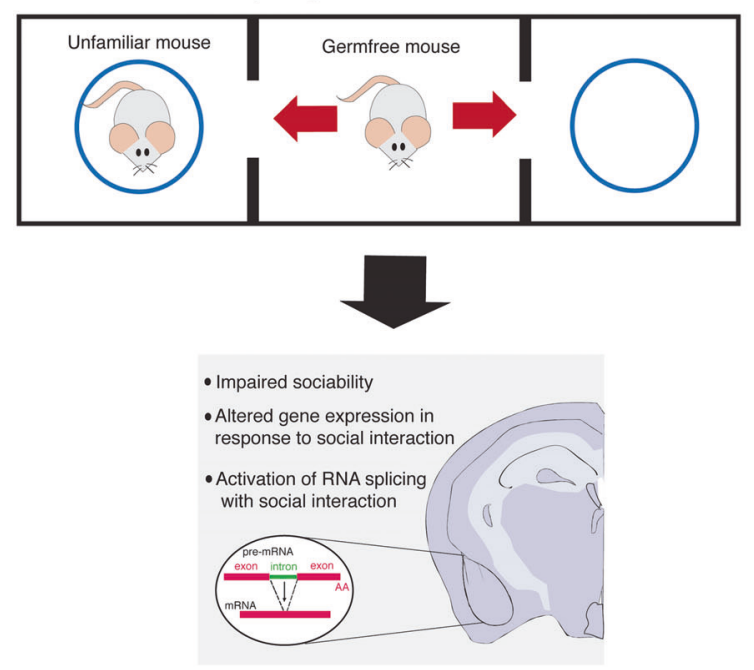

${ }^{1}$ Department of Anatomy and Neuroscience, University College Cork, Cork, Ireland; ${ }^{2}$ APC Microbiome Institute Ireland, University College Cork, Cork, Ireland and ${ }^{3}$ Department of Psychiatry and Neurobehavioral Science, University College Cork, Cork, Ireland

Correspondence: John F. Cryan (j.cryan@ucc.ie)

Received: 14 August 2018 Accepted: 23 September 2018

Published online: 19 October 2018 
Fig. 1 Altered social behaviour-induced changes in the amygdala. Under baseline activity the amygdala of germ-free mice is an activated state. Transcriptomic analysis demonstrated an upregulation of several immediate early response genes such as Fos, Fosb, Egr2 or $\mathrm{Nr} 4 \mathrm{a} 1$ in association with increased CREB signalling in GF mice (see [5] for full details). Moreover, when a germ-free mouse is introduced to a social stimulus the normal transcriptional pathway recruitment is absent but instead genes involved in alternative splicing are enriched (see [6] for full details of genes affected)

However, GF mice displayed a strikingly different pattern of amygdala gene activity in response to social interaction [6] (Fig. 1). In particular, the dynamic, stimulus-dependent transcriptional regulation seen in controls was attenuated and replaced by a marked increase in expression of splicing factors and alternative exon usage. This reveals a potential molecular basis for how the host microbiome is crucial for a normal behavioural response during social interaction. Moreover, social behaviour was correlated with the amygdala gene-expression response. These results reveal one of the key steps leading from absence of bacteria during brain development to a phenotype associated with reduced sociability in adulthood in mice. These data thus enhance our understanding of the link between the microbiome and brain health and neurodevelopmental disorders such as autism spectrum disorders. Future studies will be needed to determine what are the exact microbial signals that regulate alternative splicing events in the amygdala and whether they can be harnessed for therapeutic benefit.

\section{FUNDING AND DISCLOSURE}

The authors are supported by Science Foundation Ireland (Grant Nos. SFI/12/RC/2273). They have had research support from
Mead Johnson, Cremo, 4D Pharma, Suntory Wellness, Dupont and Nutricia.

\section{ACKNOWLEDGEMENTS}

The authors would like to thank Dr. Roman Stilling for his stimulating insights into amygdala-microbiome interactions that form the basis of this short article and Dr. Stilling and Dr. Kiran Sandhu for assistance with Fig. 1.

\section{ADDITIONAL INFORMATION}

Publisher's note: Springer Nature remains neutral with regard to jurisdictional claims in published maps and institutional affiliations.

\section{REFERENCES}

1. Allen AP, Dinan TG, Clarke G, Cryan JF. A psychology of the human brain-gutmicrobiome axis. Soc Personal Psychol Compass. 2017;11:e12309.

2. Desbonnet L, Clarke G, Shanahan F, Dinan TG, Cryan JF. Microbiota is essential for social development in the mouse. Mol Psychiatry. 2014;19:146-8.

3. Cowan CSM, Hoban AE, Ventura-Silva AP, Dinan TG, Clarke G, Cryan JF. Gutsy moves: the amygdala as a critical node in microbiota to brain signaling. Bioessays. 2018:40:1700172.

4. Luczynski P, McVey Neufeld KA, Oriach CS, Clarke G, Dinan TG, Cryan JF. Growing up in a bubble: using germ-free animals to assess the influence of the gut microbiota on brain and behavior. Int J Neuropsychopharmacol. 2016;19:pii: pyw020.

5. Stilling RM, Ryan FJ, Hoban AE, Shanahan F, Clarke G, Claesson MJ, et al. Microbes \& neurodevelopment-absence of microbiota during early life increases activity-related transcriptional pathways in the amygdala. Brain Behav Immun. 2015;50:209-20.

6. Stilling RM, Moloney GM, Ryan FJ, Hoban AE, Bastiaanssen TF, Shanahan F, et al. Social interaction-induced activation of RNA splicing in the amygdala of microbiome-deficient mice. eLife. 2018;7:pii: e33070.

\title{
Novel models of drug relapse and craving after voluntary abstinence
}

\author{
Marco Venniro ${ }^{1}{ }^{1}$, Daniele Caprioli ${ }^{2,3}$ and Yavin Shaham ${ }^{1}$ \\ Neuropsychopharmacology (2019) 44:234-235; https://doi.org/10.1038/s41386-018-0196-4
}

Relapse to drug use during abstinence is a core feature of addiction. Since the 1980s, this clinical scenario has been studied using animal models where relapse is assessed after experimenterimposed cessation of drug self-administration by either extinction of the drug-reinforced responding or homecage forced abstinence [1]. However, despite strides toward understanding neuronal mechanisms of relapse in these models, treatment options remain largely unchanged. One potential reason for this state-of-affairs is that animal models of addiction rarely incorporate voluntary aspects of human abstinence, which often occurs due to the availability of alternative nondrug rewards (e.g., employment and supportive social environment). This is exemplified in contingency management where nondrug rewards (monetary vouchers), given in exchange for being drug-free, can maintain abstinence for many months. However, when contingency management discontinues, most drug users relapse.

Based on these considerations, we introduced a contingency management-based relapse model, where we achieve long-lasting voluntary abstinence prior to the relapse tests by giving rats mutually exclusive choices between a drug and palatable food [2]. In the initial study, we trained male rats to self-administer the food and methamphetamine in established addiction models-escalation and DSM-IV-based-and found that rats will voluntarily abstain from drug self-administration for at least 3 weeks and then show incubation of methamphetamine craving (time-dependent increases in drug seeking during abstinence) [2]. Subsequently, we

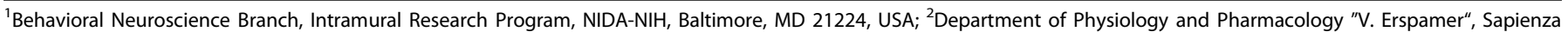
University of Rome, Rome, Italy and ${ }^{3}$ Santa Lucia Foundation (IRCCS Fondazione Santa Lucia), Rome, Italy

Correspondence: Marco Venniro (venniro.marco@nih.gov)

Published online: 11 September 2018 